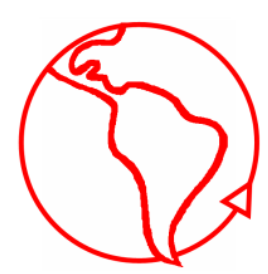

\title{
La mirada de la autopoiesis respecto de la constitución
}

\author{
The View of Autopoiesis with regard to the Constitution
}

Germano Schwartz

Centro Universitario La Salle (Unilasalle), Brasil

\section{RESUMEN}

En el presente ensayo se pretende -al encontrar evidencias de cuestionamientos acerca de la constitución como la norma superior del ordenamiento jurídico- estudiar la ley mayor desde otra base de análisis i.e. la sistémico-autopoiética, ante la insuficiencia de los modelos jurídicos tradicionales respecto de la posición de una ley fundamental en el contexto de la sociedad actual. El presente trabajo parte de la premisa de que la constitución solamente puede ser comprendida desde la diferenciación funcional entre el sistema político y el sistema jurídico, representando el acoplamiento estructural entre ambos sistemas. En este sentido, la autopoiesis de la constitución opera en virtud de su autoreferencialidad, contando al mismo tiempo con una apertura hacia los otros subsistemas. La jerarquía constitucional kelseniana ya no responde a los anhelos de una sociedad de riesgo y de indeterminación. La circularidad decisional adapta y transforma la constitución a partir de sus propios elementos jurídicos y con base en una nueva lógica, más apta para responder a las influencias comunicacionales de los demás subsistemas sociales. En este razonamiento, la superioridad de la constitución y su carácter de ley fundamental no son insumos para una definición estática. Tales características son (re)construidas en el interior del sistema a partir de su lógica interna propia.

PALABRAS ClAVE: Sistemas sociales; Sistema jurídico; Constituciones; Autopoiesis

\section{ABSTRACT}

The present essay claims -on having found evidence of questions about the constitution as the highest norm of the juridical arranging- for a study of the superior law from another point of view i.e., the autopoietic-systemic, given the inadequacy of the traditional juridical models regarding the position of a fundamental law in the context of current society. This essay starts from the premise that constitution can be understood only based on functional differentiation between political and legal system, representing this the structural coupling between both systems. In this sense, the autopoiesis of constitution operates through self-reference, having at the same time an openness to other subsystems. Kelsen's constitutional hierarchy no longer responds to the aspirations of a risk and uncertainty society. The decisional circularity adapts and transforms the constitution from its own legal elements based on a new logic, more suitable to answer to the communication influences of other social subsystems. In this line of reasoning, the superiority of the constitution and its character of fundamental law are not information for a static definition. Such features are (re)constructed inside the system from its own internal logic.

KEYWORDS: Social systems; Legal system; Constitutions, Autopoiesis

REVISTA MAD - UNIVERSIDAD DE CHILE

Revista del Magíster en Análisis Sistémico Aplicado a la Sociedad

Facultad de Ciencias Sociales, Departamento de Antropología.

Universidad de Chile

www.revistamad.uchile.cl 


\section{INTRODUCCIÓN}

Una de las grandes cuestiones de los tiempos actuales, en el derecho, está en la posición de la constitución como ápice de un ordenamiento jurídico. Es emblemático el ejemplo de la construcción de las decisiones del Tribunal de Justicia Europeo que frecuentemente se vienen sobreponiendo a las cartas magnas de los estados-nación pertenecientes a esa asociación de países. No se deben olvidar también las decisiones oriundas de organismos internacionales como por ejemplo OMC y FMI. Muchas de ellas contradicen las leyes fundamentales de algunos países - sobre todo los periféricos -, obligándolos a reformarlas o desobedecerlas, teniendo en vista el poder económico coercitivo ejercido por aquellos grupos. Se comprueba, pues, una ausencia de soberanía, elemento esencial para el entendimiento de la constitución como símbolo de independencia de un estado (puebloterritorio-gobierno).

De hecho, se observa además la insuficiencia de los modelos jurídicos tradicionales para responder acerca de la posición de una ley fundamental en el contexto de la sociedad actual. En este sentido, aquí se busca estudiar la ley mayor desde un nuevo tipo de observación: la sistémicoautopoiética.

\section{1.}

La idea de la constitución como elemento perteneciente única y exclusivamente al sistema jurídico solamente puede ser abordada desde una idea sistémico-autopoiética, o sea, esta idea solo surge cuando se excluye la concepción de la unidad entre derecho y política que vigoraba en los siglos XVIII y XIX, posteriormente rechazada por Hans Kelsen (Warat 1983). La constitución solamente puede ser comprendida desde la diferenciación funcional entre el sistema político y el sistema jurídico (Luhmann 1995: 106), puesto que se presenta como una "adquisición evolutiva" de la sociedad, sustituyendo el derecho natural por el derecho de la razón, tornándolos operacionalizables (De Giorgi 1998: 118s.).

En ese sentido, la adquisición evolutiva de la constitución puede ser sugerida como un proceso inter-organizativo que puede venir a desencadenar un texto constitucional más próximo a los estadios societarios actuales. Este es el caso, por ejemplo, de la Comunidad Europea y del propio Mercosur. La necesidad de integración, demandada por el sistema social, 
forzará a la constitución a adaptarse y a ser (re)creada. Esa idea es bien explicada por José Canotilho:

Pero Luhmann continuó después abordando algunas cuestiones constitucionales, además de las cuestiones teóricas que están siempre en el centro de su pensamiento. Fue apuntando hacia la idea de una constitución evolutiva, porque esta era una idea interesante a nivel de inter-organizatividad. Y esta es una de las premisas básicas de Luhmann, ver en la idea de constitución evolutiva una posibilidad de la propia evolución del constitucionalismo europeo. No es por casualidad que el Tribunal de Justicia de la Comunidad Europea empezó a hablar (antes de tiempo, a mi juicio) de "Constitución Europea", sin existir un poder constituyente para crearla. (Canotilho 2003: 23)

Luhmann ve en la constitución, por lo tanto, algo dinámico, separado de las tradicionales concepciones del Estado que posibilitaban el mantenimiento temporal de una constitución. Y eso solamente es alcanzado, paradójicamente, por su aislamiento con relación a los demás sistemas, pues solamente de esa manera la constitución consigue un nivel tal de organización, que le posibilita avanzar en dirección al futuro. Además, la diferenciación entre constitución y política tiene una serie de ventajas, precisadas por Pilar Alcóver (1993: 344):

1) Si en el sistema jurídico, y también en el sistema político, las decisiones programadas y las programables están diferenciadas y son atribuidas a diversos órganos del Estado, esa organización permite una mayor racionalidad en la división de tareas y, con ello, la separación de las responsabilidades por el mantenimiento o modificación de los programas, a partir de su relación con el entorno;

2) También permite separar la coercibilidad, el uso del monopolio de la fuerza física y potenciar aquella en detrimento de esta. Permite, también, separar las formas utilizadas por el sistema jurídico para proteger contemporáneamente la seguridad de las expectativas normativas y su adaptación a la realidad;

3) La especificación funcional del derecho no impide la observación de la importancia de las funciones desempeñadas por determinadas instituciones y normas jurídicas para el propio mantenimiento de la diferenciación funcional de la sociedad (este es el caso, por ejemplo, de los derechos fundamentales - derecho fundamental a la salud). 
De este modo, la mirada desde el prisma de la teoría de los sistemas no implica que la constitución no mantiene contacto con los demás subsistemas sociales. De hecho, es el "acoplamiento estructural" entre derecho y política (Luhmann 1995: 118) donde se da por excelencia la comunicación del sistema jurídico con el entorno. Este acoplamiento queda claro cuando se reconoce, por ejemplo, que la democratización de la política (la codificación: gobierno/oposición) "exige, finalmente, todavía más protección jurídica al particular, en especial en lo concerniente a sus derechos constitucionales" (Luhmann 2001: 21). La diferencia es que, cuando decida el sistema del derecho, deberá haber justicia para las decisiones suministradas por su código propio (derecho/no derecho). Ahora los políticos no deben interpretar la constitución, sino que deben cumplirla, pues el objeto de su ciencia es distinto de la especialidad jurídica (Hespanha 1999: 60).

En el mismo sentido, la constitución utiliza conceptos políticos tales como pueblo, elector, partidos políticos y estado y, con ello, remite al sistema político. Sin embargo, cuando dichos conceptos son positivados en un texto constitucional, estos pasan a ser analizados/investigados como derecho y así serán operacionalizados. La lección de Raffaele de Giorgi sobre esto es esclarecedora:

Mediante la constitución "el derecho reacciona a su autonomía", en la medida en la que dispone de clausura y, por consiguiente, de autocontrol. Por otro lado, la política garantiza su independencia y puede contener las presiones involutivas de los estratos y canalizar las imposiciones de los privilegios. La constitución "cierra el sistema jurídico porque lo regula como un ámbito en el cual ella misma reaparece": la constitución es derecho que trata de la conformidad del derecho consigo mismo. (De Giorgi 1998: 119)

\section{2.}

Lo anterior implica que la autopoiesis constitucional está basada en su auto-referencialidad. En el momento de la operación jurídica, que toma como base la constitución, el derecho produce sentido a partir de su propia unidad distintiva y sus propias especificidades. No se confunde la autoreferencia producida por el sistema político en relación con la constitución, con la self-reference constitucional del sistema jurídico, pues, como señala Benedito Hespanha (1999: 60), son sistemas distintos en los cuales se incluyen las referencias de las personas o de los grupos que las experimentan. 
Sin embargo, es esa misma distinción auto-referencial la que ofrece a la constitución la posibilidad de una apertura exógena y cognitiva al entorno que la rodea (Luhmann 1995: 125). En esa dinámica permanente de comunicación con los demás subsistemas sociales, la constitución se va autorregulando y, cada vez más, distinguiéndose del exterior, formulando una unidad referencial propia de estructuras, principios y operaciones específicas.

De este modo, los principios constitucionales son pre-requisitos de decisión y no condiciones de justicia. Son esquemas operativos de naturaleza condicional, limitados por la función estructural dada por el sistema en el cual están incluidos (el derecho: decisión). Las precondiciones de los principios son proporcionadas por la diferenciación funcional, pues es ella la que permite la individualización de los modos de comportamiento.

Junto con la individualización de los principios mediante la diferenciación, la generalización de las expectativas normativas por medio de la constitución, se convierte en base y requisito estructural del derecho. Como sostiene De Giorgi, "ambos son garantizados y estabilizados a través del derecho y en el sistema del derecho, por los principios constitucionales". (De Giorgi 1998: 118).

En esa línea, para ejemplificar, el principio constitucional brasileño del derecho a la salud (art. 196, de la $\mathrm{CF} / 88$ ) es una estructura autoreferente que transforma el derecho a la salud desde el propio derecho a la salud. Garantiza la generalización y la individualización de la protección sanitaria en el sistema jurídico, posibilitando al mismo tiempo la apertura externa y la clausura interna del derecho.

\section{3.}

De ello resulta que la idea de constitución es paradójica. La positividad y la operatividad interna de la constitución son posibilitadas por su necesaria apertura cognoscitiva a los demás subsistemas funcionales de la sociedad. Dicho de otra forma, la positividad constitucional es nada más que la expresión de autodeterminación del sistema jurídico.

Adicionalmente, la propia constitución, cuando se auto-observa, refuerza la idea de entrecruzamiento auto-referencial de sus partes componentes. Los principios conducen a los derechos fundamentales, los que se conectan a su vez con la organización del estado. La organización estatal está vinculada a la organización de los poderes. Mediante un movimiento 
cíclico-recursivo, se produce la auto-referencia que posibilita la intracomunicación y la intra-producción constitucional.

Después de su movimiento intra-recursivo, la constitución se comunica, mediante decisiones, con las demás normas y estructuras componentes del sistema jurídico, de tal forma que las normas de derecho reproducen otras normas de derecho, en el contexto de las propias relaciones del sistema constitucional (Hespanha 1999: 60). Empleando el concepto de Gunther Teubner (1989), se podría decir que la constitución se constituye en parte integrante del sistema autopoiético de segundo orden denominado derecho (Hespanha 1999: 61).

Por otro lado, cuando la constitución, luego de su auto-referencia, se pone en movimiento e influye en los demás subsistemas sociales, se contacta especialmente con el subsistema político. Tómese como ejemplo el caso de la salud en Brasil: en el sistema jurídico se debe decidir con base en el código derecho/no-derecho, bajo el fundamento proporcionado por el artículo 196 de la constitución. En el sistema político se debe tomar la decisión de direccionar los recursos públicos para fines sanitarios, de acuerdo con los programas políticos elaborados desde la unidad distintiva gobierno/oposición. Dicho de otra forma: la constitución, en el lenguaje de Teubner (1989), se hace presente y actuante en los demás subsistemas sociales por fuerza de su inter-legalidad. Así, la constitución puede ser observada como el locus de construcción de lo nuevo, a partir de las descripciones ofrecidas por los demás subsistemas, en los cuales actúa como factor condicionante de decisión comunicacional, y en el interior de subsistemas funcionales y diferenciados, así como en el medio de la comunicación, establecido en el entorno del sistema social, donde se incluye la diferenciación sistema/entorno.

En este sentido, una nueva realidad sanitaria pasa también por la autopoiesis constitucional que establece el derecho a la salud. Como afirma Hespanha: "la autopoiesis del sistema constitucional hace concreta la construcción positiva de la juridicidad de los principios, las reglas y las instituciones que regulan lo político por medio de un proceso abierto a la sociedad." (Hespanha 1999: 75)

\section{4.}

La decisión nuevamente toma lugar de relieve. La cuestión es quién debe decidir sobre la constitución, dándole continuidad y efectividad. Si la ley y la jurisprudencia actúan en la decisión judicial, se observa que el problema 
es diferente en los grados "superior" e "inferior" de las leyes (Luhmann 1990: 157). Como ya se ha señalado, la tarea decisoria sobre la carta magna le cabe al órgano constitucional del poder judicial. Con ello, la intrasuperioridad circular y central de la constitución está dada por el hecho que los tribunales responsables son la última escala de la jurisdicción, donde se cierra el sistema y no hay más posibilidad de buscar otra decisión.

A pesar de lo anterior, no se excluye la hipótesis de que la legislación pueda presentar una no conformidad con la constitución, más aún porque la dinámica acto-norma-acto hace que las decisiones de los tribunales constitucionales reafirmen que "todo el derecho puede estar de acuerdo con, o contrario a, la constitución" (Luhmann 1990: 158). Son las decisiones de cuño constitucional las que dan continuidad a la apertura de la constitución, o, como quiere Canotilho (2003), la constitucionalización fundamental de la sociedad ${ }^{1}$.

De esto resulta la necesidad de una organización interna al sistema jurídico que pugne por la observancia y respeto de la constitución. Luego, es coherente con el pensamiento luhmanniano la noción de un control de constitucionalidad que filtre y verifique las normas legales permisibles. De este modo, autopoieticamente, "un sistema debe crear por si mismo un complejo de normas de control formal, por ejemplo en la forma de una constitución que regula el procedimiento y proporciona una preselección abstracta de normas legales permisibles". (Luhmann 1996: 115).

En este sentido, cuando Luhmann habla de preselección abstracta de normas, hay una evidente conexión con el control concentrado de constitucionalidad, donde se produce el examen de (in)constitucionalidad de una ley que es dirigida a todos, para el caso en cuestión y hecha de forma abstracta. En el caso brasileño, este control es de competencia del Supremo Tribunal Federal, ya sea por medio de Acción Directa de Inconstitucionalidad, por Acción Declaratoria de Constitucionalidad, o, inclusive, por Acción de Incumplimiento de Precepto Fundamental.

El legislador debe combatir la corrupción de la constitución (Luhmann 1995: 106), a pesar que sea su inobservancia la que la reafirme como ley fundamental. Corrupción en el sentido de ser violada. Esta idea debe partir del propio proceso legislativo que debe producir normas conforme a

\footnotetext{
${ }^{1}$ En exposición proferida en el día 18/10/2002, en la PUCRS, el Profesor José Joaquim Gomes Canotilho defendió la idea de que las teorías de la diferenciación posibilitan a la constitución la comunicación con los demás sistemas sociales. En el mundo globalizado, el profesor defiende que al revés de una economización fundamental, ocurre una constitucionalización fundamental, diseminando los valores constitucionales a los más lejanos cuadrantes de la Tierra.
} 
la carta magna, a fin de que la recursividad del derecho sea afirmada y, así, se torne posible su apertura al entorno. Dicho de otra manera: todo el derecho está sujeto al examen de constitucionalidad (Luhmann 1995: 118).

Siguiendo esta línea, también debe haber una decisión anterior de constitucionalidad (Luhmann 2001: 25), o sea, el equivalente a las Comisiones de Constitución y Justicia existentes en cada casa del Poder Legislativo brasileño. Aunque tomadas de otro sistema, esta operación es justiciable, puesto que la decisión se da con base en la fórmula derecho (constitución)/ no derecho (no constitución).

\section{5.}

Por otro lado, la constitución (y su función), así como todo lo demás, depende del observador. Para el sistema político, esta se vería apenas como una pre-condición decisoria establecida por el poder constituyente originario, o sea, como un medio programático para la adopción de decisiones políticas. La observación hecha por un tribunal, en cambio, debe tener en cuenta su papel de asegurar expectativas normativas. De esta manera, el poder judicial no debe ejercer el papel de fiscal del poder constituyente originario; debe ser, en cambio, el guardián de la constitución (Campilongo 2000: 86), mediante el programa (legislación) ofrecido por el poder legislativo pero diferenciándose de él, de tal forma que se reconstruya el sentido de la constitución.

Dicho de otra forma: para ambos sistemas (político y jurídico), la constitución captura la influencia del entorno (Luhmann 2001: 26). Para el sistema político, la constitución acarrea la legitimación ordenadora de sus actos, una regulación que lo vincula. Para el sistema jurídico, la constitución aumenta la posibilidad, por intermedio del derecho, de la concretización de las políticas públicas allí enunciadas. Como señala Evaristo Navarro:

La constitución, a modo de ejemplo, es una estructura presente con características distintas, tanto en el sistema político como en el jurídico. En ambos cumple la misión de introducir el entorno en el sistema a través de la autorreferencia. El sistema político, mediante la interpretación que lleva a cabo de los textos constitucionales, se representa la ilusión de un acoplamiento y una regulación ordenadora del derecho en sus asuntos internos... Por su parte, el sistema jurídico, a través de la constitución, se ve confrontado con la necesidad de elaborar de continuo las iniciativas políticas que se presentan. Paralelamente, se incrementan sus posibilidades de presentar estas iniciativas políticas en forma jurídica. (Navarro 1998: 123s.) 
La constitución es, por lo tanto, el medium, el acoplamiento estructural de la política y del derecho. En esta interpretación, la constitución formula (v. en el caso brasileño, cláusulas pétreas y el procedimiento legislativo establecidos en el art. 59 y siguientes de la CF/88) el modo por el cual se modifica y el método que el poder legislativo posee para modificar la norma. Con ello, se juridizan relaciones políticas y se intermedian razones políticas de transformación de la norma jurídica (Guerra 2001: 194).

\section{Conclusiones}

De los argumentos expuestos se puede concluir que la jerarquía constitucional kelseniana ya no responde a los anhelos de una sociedad de riesgo y de indeterminación (Luhmann 1995: 113s.). La circularidad decisional adapta y transforma la constitución a partir de sus propios elementos jurídicos y con base en una nueva lógica, más apta para responder a las influencias comunicacionales de los demás subsistemas sociales.

De esta forma, el derecho puede también ser observado como la unidad de la diferencia entre el derecho constitucional y el derecho restante (Luhmann 1995: 114). El derecho está orientado conforme a la constitución. Como ya se ha señalado, o está de acuerdo o en desacuerdo con el texto constitucional. En la primera hipótesis, la auto-referencialidad sigue su ciclo normal y las decisiones de carácter constitucional atraviesan el sistema, reconstruyéndolo. En el segundo caso, también sucederá la autopoiesis pero de forma negativa: lo que está en desacuerdo con la constitución reafirma el derecho por no ser este derecho.

En este razonamiento, la superioridad de la constitución y su carácter de ley fundamental no son insumos para una definición estática. Tales características son (re)construidas en el interior del sistema a partir de su lógica interna propia. Significa, como apunta Luhmann, "que la inmutabilidad, vulnerabilidad, el carácter de valor supremo, etc., deben estar integrados en el propio sistema del derecho" (Luhmann 1995: 112). En esta senda, las características de la constitución, en un sistema autopoiético, nos llevan a algunas consideraciones (Luhmann 1995: 116):

1) Es la constitución, por intermedio de sus principios y normas, la que posibilita su propia auto-referencia;

2) con eso hay simetría infraconstitucional desde la asimetría interna del texto fundamental; 
3) la constitución regula la producción del derecho y ella misma prevé su revisión, actualizando las normas inferiores y a ella misma;

4) la constitución hace posible la distinción entre derecho constitucional y el resto del derecho;

5) la constitución no depende del sistema político en el momento de su aplicación desde el sistema jurídico, sin embargo recibe su influencia en el momento de su creación;

6) de ello resulta que la autopoiesis jurídico-constitucional necesita su auto-referencialidad para su (re)creación constante;

7) finalmente, el fundamento de validez de la constitución implica únicamente la necesidad de dar a la constitución una unidad sistémica que le posibilite (re)crearse desde la distinción sistema/entorno, dentro del sistema social. RM

\section{REFERENCIAS}

Alcover, P. (1993). El derecho en la teoría de la sociedad de Niklas Luhmann. Barcelona: J.M. Bosch Editor.

Campilongo, C. (2000). O direito na sociedade complexa. São Paulo: Max Limonade.

Canotilho, J. (2003). $1^{\text {a }}$ Parte - Videoconferência - 21/02/02 - UFPR. En J. Coutinho (Ed.), Canotilho e la Constituição Dirigente. Rio de Janeiro: Renovar.

De Giorgi, R. (1998). Direito, democracia e risco: vínculos com o futuro. Porto Alegre: SAFE.

Guerra, W. (2001). Teoria da ciencia jurídica. São Paulo: Saraiva.

Hespanha, B. (1999). A autopoiese na construção do jurídico e do político de um sistema constitucional. Cadernos de Direito Constitucional e Ciência Política, 27, $59-78$.

Luhmann, N. (1990). La posição dos tribunais no sistema jurídico. Revista da Ajuris, Porto Alegre, 17(49), 149-168. [Traduzido por Peter Naumann e revisado pela Prof. Vera Jacob de Fradera]

Luhmann, N. (1996). Confianza. Barcelona: Anthropos.

Luhmann, N. (1995). La Constitution comme Acquis Évolutionnaire. Droits - Revue Française de Théorie Juridique, 22, 102-125.

Luhmann, N. (2001). Poder, política y derecho. Metapolítica, 5(20), 11-39.

Navarro, E. (1998). El derecho y la moderna teoría de sistemas. En J. Domínguez \& N. Ulgar (Eds.), La joven sociología jurídica en España: Aportaciones para una consolidación. Oñati Papers - 6. Oñati: IISJ.

Teubner, G. (1989). O direito como sistema autopoiético. Lisboa: Calouste Gulbenkian.

Warat, L. (1983). La Pureza do Poder. Santa Catarina: UFSC. 
SOBRE EL AUTOR

Germano Schwartz es Doctor en Derecho por la Universidade do Vale do Rio dos Sinos, Brasil. Profesor de la Escuela de Derecho de la Facultad de Serra Gaúcha (FSG), Brasil. Coordinador de la Maestría en Derecho del Centro Universitario La Salle (Unilasalle), Brasil. Secretario del comité de investigación sobre sociología del derecho de la Asociación Internacional de Sociología (ISA). Segundo Vice-Presidente de la Asociación Brasileña de Investigadores en Sociología del Derecho. Entre sus publicaciones destacan: $A$ Verdade sobre a Autopoiese no Direito (Porto Alegre, 2009), Direito e Literatura. O Encontro entre Themis e Apolo (Curitiba, 2008), Comunicação e Direito: Semiótica, Literatura e norma. Porto Alegre, 2008).

CONTACTO: germano.schwartz@globo.com

Recibido: Diciembre 2014

Aceptado: Enero 2015 